\title{
A note on Fourier coefficients of Poincaré series
}

\section{Journal Article}

\section{Author(s):}

Kowalski, Emmanuel; Saha, Abhishek; Tsimerman, Jacob

Publication date:

2011-01

Permanent link:

https://doi.org/10.3929/ethz-b-000042274

\section{Rights / license:}

In Copyright - Non-Commercial Use Permitted

\section{Originally published in:}

Mathematika 57(1), https://doi.org/10.1112/S0025579310001804 


\section{A NOTE ON FOURIER COEFFICIENTS OF POINCARÉ SERIES}

\section{EMMANUEL KOWALSKI, ABHISHEK SAHA AND JACOB TSIMERMAN}

Abstract. We give a short and "soft" proof of the asymptotic orthogonality of Fourier coefficients of Poincaré series for classical modular forms as well as for Siegel cusp forms, in a qualitative form.

§1. Introduction. The Petersson formula (see, e.g., [2, Ch. 14]) is one of the most basic tools in the analytic theory of modular forms on congruence subgroups of $\operatorname{SL}(2, \mathbf{Z})$. One of its simplest consequences, which explains its usefulness, is that it provides the asymptotic orthogonality of distinct Fourier coefficients for an orthonormal basis in a space of cusp forms, when the analytic conductor is large (e.g., when the weight or the level is large). From the proof of the Petersson formula, we see that this orthonormality is equivalent (on a qualitative level) to the assertion that the $n$th Fourier coefficient of the $m$ th Poincaré series is essentially the Kronecker symbol $\delta(m, n)$.

In this note, we provide a direct "soft" proof of this fact in the more general context of Siegel modular forms when the main parameter is the weight $k$. Although this is not sufficient to derive the strongest applications (e.g., to averages of $L$-functions in the critical strip), it provides at least a good motivation for the more quantitative orthogonality relations required for those. And, as we show in our paper [4] concerning the local spectral equidistribution of Satake parameters for certain families of Siegel modular forms of genus $g=2$, the "soft" proof suffices to derive some basic consequences, such as the analogue of "strong approximation" for cuspidal automorphic representations, and the determination of the conjectural "symmetry type" of the family. See Corollary 3 for a simple example of this when $g=1$.

§2. Classical modular forms. In this section, we explain the idea of our proof for classical modular forms; we hope this will be useful as a comparison point in the next section, especially for readers unfamiliar with Siegel modular forms. Let $k \geq 2$ be an even integer, $m \geq 1$ an integer. The $m$ th Poincaré series of weight $k$ is defined by

$$
P_{m, k}(z)=\sum_{\gamma \in \Gamma_{\infty} \backslash \Gamma}(c z+d)^{-k} e(m \gamma \cdot z),
$$


where $\Gamma=\operatorname{SL}(2, \mathbf{Z})$, acting on the Poincaré upper half-plane $\mathbf{H}$,

$$
\Gamma_{\infty}=\left\{ \pm\left(\begin{array}{cc}
1 & n \\
0 & 1
\end{array}\right): n \in \mathbf{Z}\right\}
$$

is the stabilizer of the cusp at infinity, and we write

$$
\gamma=\left(\begin{array}{ll}
a & b \\
c & d
\end{array}\right), \quad(a, b, c, d) \in \mathbf{Z}^{4} .
$$

It is well known that for $k \geq 4, m \geq 1$, this series converges absolutely and uniformly on compact sets, and that it defines a cusp form of weight $k$ for $\Gamma=\operatorname{SL}(2, \mathbf{Z})$. We denote by $p_{m, k}(n), n \geq 1$, the Fourier coefficients of this Poincaré series, so that

$$
P_{m, k}(z)=\sum_{n \geq 1} p_{m, k}(n) e(n z)
$$

for all $z \in \mathbf{H}$.

PROPOSITION 1 (Asymptotic orthogonality of Fourier coefficients of Poincaré series). With notation as above, for fixed $m \geq 1, n \geq 1$, we have

$$
\lim _{k \rightarrow+\infty} p_{m, k}(n)=\delta(m, n) .
$$

Proof. The idea is to use the definition of Fourier coefficients as

$$
p_{m, k}(n)=\int_{U} P_{m, k}(z) e(-n z) d z
$$

where $U$ is a suitable horizontal interval of length one in $\mathbf{H}$, and $d z$ is the Lebesgue measure on such an interval; we then let $k \rightarrow+\infty$ under the integral sign, using the definition of the Poincaré series to understand that limit.

We select

$$
U=\left\{x+i y_{0}:|x| \leq 1 / 2\right\}
$$

for some fixed $y_{0}>1$. The Lebesgue measure is then of course $d x$.

Consider a term

$$
(c z+d)^{-k} e(m \gamma \cdot z)
$$

in the Poincaré series as $k \rightarrow+\infty$. We have

$$
\left|(c z+d)^{-k} e(m \gamma \cdot z)\right| \leq|c z+d|^{-k}
$$

for all $z \in \mathbf{H}$ and $\gamma \in \operatorname{SL}(2, \mathbf{Z})$, since $m \geq 0$ and $\gamma \cdot z \in \mathbf{H}$. But for $z \in U$, we find

$$
|c z+d|^{2}=(c x+d)^{2}+c^{2} y_{0}^{2} \geq c^{2} y_{0}^{2} .
$$

If $c \neq 0$, since $c$ is an integer, the choice of $y_{0}>1$ leads to $c^{2} y_{0}^{2}>1$, and hence

$$
\left|(c z+d)^{-k} e(m \gamma \cdot z)\right| \rightarrow 0
$$

as $k \rightarrow+\infty$, uniformly for $z \in U$ and $\gamma \in \Gamma$ with $c \neq 0$. On the other hand, if $c=0$, we have $\gamma \in \Gamma_{\infty}$; this means that it corresponds to a single term, which 
we take to be $\gamma=\mathrm{Id}$, and we then have

$$
(c z+d)^{-k} e(m \gamma \cdot z)=e(m z)
$$

for all $k$ and $z \in U$.

Moreover, all this shows also that

$$
\left|(c z+d)^{-k} e(m \gamma \cdot z)\right| \leq|c z+d|^{-4}
$$

for $k \geq 4$ and $\gamma \in \Gamma_{\infty} \backslash \Gamma$. Since the right-hand side converges absolutely and uniformly on compact sets, we derive by dominated convergence that

$$
P_{m, k}(z) \rightarrow e(m z)
$$

for all $z \in U$. The above inequality gives, further,

$$
\left|P_{m, k}(z)\right| \leq \sum_{\gamma \in \Gamma_{\infty} \backslash \Gamma}|c z+d|^{-4}
$$

for $k \geq 4$ and $z \in U$. Since $U$ is compact, we can integrate by dominated convergence again to obtain

$$
\int_{U} P_{m, k}(z) e(-n z) d z \rightarrow \int_{U} e((m-n) z) d z=\delta(m, n)
$$

as $k \rightarrow+\infty$.

It turns out that the same basic technique works for the other most important parameter of cusp forms, the level. Now for $q \geq 1$ and $m \geq 1$ integers, let

$$
P_{m, q}(z)=\sum_{\gamma \in \Gamma_{\infty} \backslash \Gamma_{0}(q)}(c z+d)^{-k} e(m \gamma \cdot z)
$$

be the $m$ th Poincare series of weight $k$ for the Hecke group $\Gamma_{0}(q)$, and let $p_{m, q}(n)$ denote its Fourier coefficients.

PROPOSITION 2 (Orthogonality with respect to the level). With notation as above, for $k \geq 4$ fixed, for any fixed $m$ and $n$, we have

$$
\lim _{q \rightarrow+\infty} p_{m, q}(n)=\delta(m, n) .
$$

Proof. We start with the integral formula

$$
p_{m, q}(n)=\int_{U} P_{q, m}(z) e(-n z) d z
$$

as before. To proceed, we observe that $\Gamma_{\infty} \backslash \Gamma_{0}(q)$ is a subset of $\Gamma_{\infty} \backslash \Gamma$, and hence we can write

$$
P_{m, q}(z)=\sum_{\gamma \in \Gamma_{\infty} \backslash \Gamma} \Delta_{q}(\gamma)(c z+d)^{-k} e(m \gamma \cdot z),
$$

where

$$
\Delta_{q}\left(\left(\begin{array}{ll}
a & b \\
c & d
\end{array}\right)\right)= \begin{cases}1 & \text { if } c \equiv 0(\bmod q) \\
0 & \text { otherwise. }\end{cases}
$$


We let $q \rightarrow+\infty$ in each term of this series. Clearly, we have $\Delta_{q}(\gamma)=0$ for all $q>c$, unless $c=0$, in which case $\Delta_{q}(\gamma)=1$. Thus

$$
\Delta_{q}(\gamma)(c z+d)^{-k} e(m \gamma \cdot z) \rightarrow 0
$$

if $c \neq 0$, and otherwise

$$
\Delta_{q}(\gamma)(c z+d)^{-k} e(m \gamma \cdot z)=e(m z) .
$$

Moreover, we have obviously

$$
\left|\Delta_{q}(\gamma)(c z+d)^{-k} e(m \gamma \cdot z)\right| \leq|c z+d|^{-k},
$$

and since $k \geq 4$ this defines an absolutely convergent series for all $z$. We therefore obtain

$$
P_{m, q}(z) \rightarrow e(m z)
$$

for any $z \in U$. Finally, the function

$$
z \mapsto \sum_{\gamma \in \Gamma_{\infty} \backslash \Gamma}|c z+d|^{-k}
$$

being integrable on $U$, we obtain the result after integrating.

Here is a simple application to show that such qualitative statements are not entirely content-free.

COROLlary 3 ("Strong approximation" for GL(2)-cusp forms). Let A be the adèle ring of $\mathbf{Q}$. For each irreducible, cuspidal, automorphic representation $\pi$ of $\mathrm{GL}(2, \mathbf{A})$ and each prime $p$, let $\pi_{p}$ be the unitary, admissible representation of $\mathrm{GL}\left(2, \mathbf{Q}_{p}\right)$ that is the local component of $\pi$ at $p$. Then, for any finite set of primes $S$, as $\pi$ runs over the cuspidal spectrum of $\operatorname{GL}(2, \mathbf{A})$ unramified at primes in $S$, the set of tuples $\left(\pi_{p}\right)_{p \in S}$ is dense in the product over $p \in S$ of the unitary tempered unramified spectrum $X_{p}$ of $\mathrm{GL}\left(2, \mathbf{Q}_{p}\right)$.

Proof. This is already known, due to Serre [7] (if one uses holomorphic forms) or Sarnak [6] (using Maass forms), but we want to point out that it is a straightforward consequence of Proposition 1; for more details, see [4, Appendix]. We first recall that the part of the unitary unramified spectrum of $\operatorname{GL}\left(2, \mathbf{Q}_{p}\right)$ with trivial central character can be identified with $[-2 \sqrt{p}, 2 \sqrt{p}]$ via the map sending Satake parameters $(\alpha, \beta)$ to $\alpha+\beta$. The subset $X_{p}$ can then be identified with $[-2,2]$, and for $\pi=\pi(f)$ attached to a cuspidal primitive form unramified at $p$, the local component $\pi_{p}(f)$ corresponds to the normalized Hecke eigenvalue $\lambda_{f}(p)$.

Now the (well-known) point is that for any integer of the form

$$
m=\prod_{p \in S} p^{n(p)} \geq 1
$$

and any cusp form $f$ of weight $k$ with Fourier coefficients $n^{(k-1) / 2} \lambda_{f}(n)$, the characteristic property

$$
\left\langle f, P_{m, k}(\cdot)\right\rangle=\frac{\Gamma(k-1)}{(4 \pi m)^{k-1}} m^{(k-1) / 2} \lambda_{f}(m)
$$


of Poincaré series (see, e.g., [2, Lemma 14.3]) implies that

$$
\begin{gathered}
p_{m, k}(1)=\sum_{f \in H_{k}} \omega_{f} \lambda_{f}(m)=\sum_{f \in H_{k}} \omega_{f} \prod_{p \in S} U_{n(p)}\left(\lambda_{f}(p)\right), \\
\omega_{f}=\frac{\Gamma(k-1)}{(4 \pi)^{k-1}} \frac{1}{\|f\|^{2}},
\end{gathered}
$$

where $H_{k}$ is the Hecke basis of weight $k$ and level one, $U_{n}$ denotes Chebychev polynomials, and $\|f\|$ is the Petersson norm. Because the linear combinations of Chebychev polynomials are dense in $C\left(\left[-2 p^{1 / 2}, 2 p^{1 / 2}\right]\right)$ for any prime $p$, the fact that

$$
\lim _{k \rightarrow+\infty} p_{m, k}(1)=\delta(m, 1)= \begin{cases}1 & \text { if all } n(p) \text { are zero } \\ 0 & \text { otherwise }\end{cases}
$$

(given by Proposition 1) shows, using the Weyl equidistribution criterion, that $\left(\pi_{p}(f)\right)_{f \in H_{k}}$, when counted with weight $\omega_{f}$, becomes equidistributed as $k \rightarrow$ $+\infty$ with respect to the product of Sato-Tate measures over $p \in S$. Since each factor has support equal to $[-2,2]=X_{p}$, this trivially implies the result.

§3. Siegel modular forms. We now proceed to generalize the previous result to Siegel cusp forms; although some notation will be recycled, there should be no confusion. For $g \geq 1$, let $\mathbf{H}_{g}$ denote the Siegel upper half-space of genus $g$ :

$$
\mathbf{H}_{g}=\left\{z=x+i y \in M(g, \mathbf{C}): t^{t}=z, y \text { is positive definite }\right\},
$$

on which the group $\Gamma_{g}=\operatorname{Sp}(2 g, \mathbf{Z})$ acts in the usual way,

$$
\gamma \cdot z=(a z+b)(c z+d)^{-1}
$$

(see, e.g., [3, Ch. 1] for such basic facts; we always write

$$
\gamma=\left(\begin{array}{ll}
a & b \\
c & d
\end{array}\right)
$$

for symplectic matrices, where the blocks are themselves $g \times g$ matrices). Let $A_{g}$ denote the set of symmetric, positive-definite matrices in $M(g, \mathbf{Z})$ with integer entries on the main diagonal and half-integer entries off it. Further, let

$$
\Gamma_{\infty}=\left\{ \pm\left(\begin{array}{ll}
1 & s \\
0 & 1
\end{array}\right): s \in M(g, \mathbf{Z}), s={ }^{t} s\right\}
$$

For $k \geq 2$, even $\dagger$, and a matrix $s \in A_{g}$, the Poincaré series $\mathcal{P}_{s, k}$ is defined by

$$
\mathcal{P}_{s, k}(z)=\sum_{\gamma \in \Gamma_{\infty} \backslash \Gamma_{g}} \operatorname{det}(c z+d)^{-k} e(\operatorname{Tr}(s(\gamma \cdot z)))
$$

for $z$ in $\mathbf{H}_{g}$. This series converges absolutely and uniformly on compact sets of $\mathbf{H}_{g}$ for $k>2 g$; indeed, as shown by Maass [5, (32), Satz 1], the series

$$
\mathcal{M}_{s, k}(z)=\sum_{\gamma \in \Gamma_{\infty} \backslash \Gamma_{g}}|\operatorname{det}(c z+d)|^{-k} \exp (-2 \pi \operatorname{Tr}(s \operatorname{Im}(\gamma \cdot z))),
$$

$\dagger$ Forms of odd weight $k$ do exist if $g$ is even, but behave a little differently; we restrict the discussion here to $k$ even, for simplicity. 
which dominates it termwise, converges absolutely and uniformly on compact sets (see also [3, p. 90]; note that, in contrast with the case of $\operatorname{SL}(2, \mathbf{Z})$, one cannot ignore the exponential factor here to have convergence). The Poincaré series $\mathcal{P}_{s, k}$ is then a Siegel cusp form of weight $k$ for $\Gamma_{g}$. Therefore, it has a Fourier expansion

$$
\mathcal{P}_{s, k}(z)=\sum_{t \in A_{g}} p_{s, k}(t) e(\operatorname{Tr}(t z)),
$$

which converges absolutely and uniformly on compact subsets of $\mathbf{H}_{g}$.

THEOREM 4 (Orthogonality for Siegel-Poincaré series). With notation as above, for any fixed $s, t \in A_{g}$, we have

$$
\lim _{k \rightarrow+\infty} p_{s, k}(t)=\delta^{\prime}(s, t) \frac{|\operatorname{Aut}(s)|}{2},
$$

where the limit is over even weights $k, \delta^{\prime}(s, t)$ is the Kronecker delta for the $\operatorname{GL}(g, \mathbf{Z})$-equivalence classes of $s$ and $t$, and $\operatorname{Aut}(s)=O(s, \mathbf{Z})$ is the finite group of integral points of the orthogonal group of the quadratic form defined bys.

This result suggests a definition of the Poincare series with an additional constant factor $2 /|\operatorname{Aut}(s)|$, in which case this theorem is exactly analogous to Proposition 1. And, indeed, this is how Maass defined them [5].

Proof. We adapt the previous argument, first writing

$$
p_{s, k}(t)=\int_{U_{g}} \mathcal{P}_{s, k}(z) e(-\operatorname{Tr}(t z)) d z
$$

where $U_{g}=U_{g}\left(y_{0}\right)$ will be taken to be the (compact) set of matrices

$$
U_{g}\left(y_{0}\right)=\mathcal{U}_{g}+i y_{0} \mathrm{Id},
$$

for some real number $y_{0}>1$ to be selected later, where

$$
\mathcal{U}_{g}=\left\{x \in M(g, \mathbf{R}): x \text { symmetric and }\left|x_{i, j}\right| \leq 1 / 2, \forall 1 \leq i, j \leq g\right\},
$$

and the measure $d z$ is again Lebesgue measure.

Before proceeding, we first recall that

$$
|e(\operatorname{Tr}(s \gamma \cdot z))| \leq 1
$$

for all $s \in A_{g}, \gamma \in \Gamma_{g}$ and $z \in \mathbf{H}_{g}$. Indeed, since $s$ is a real matrix, we have

$$
|e(\operatorname{Tr}(s \gamma \cdot z))|=\exp (-2 \pi \operatorname{Tr}(s \operatorname{Im}(\gamma \cdot z))),
$$

and the result follows from the fact that

$$
\operatorname{Tr}(s y) \geq 0
$$

for any $s \in A_{g}$ and $y$ positive definite. To see the latter, we write $y={ }^{t} q q$ for some matrix $q$, and we then have

$$
s y=s^{t} q q=q^{-1} t q
$$

with $t=q s^{t} q$; then $t$ is still positive, while $\operatorname{Tr}(s y)=\operatorname{Tr}(t)$, so $\operatorname{Tr}(s y) \geq 0$. 
We then have the following Lemma†.

LEMMA 5. For any integer $g \geq 1$, there exists a real number $y_{0}>1$, depending only on $g$, such that for any $\gamma \in \Gamma_{g}$ written

$$
\gamma=\left(\begin{array}{ll}
a & b \\
c & d
\end{array}\right), \quad(a, b, c, d) \in M(g, \mathbf{Z}),
$$

with $c \neq 0$ and for all $z \in U_{g}\left(y_{0}\right)$, we have

$$
|\operatorname{det}(c z+d)|>1
$$

whereas if $c=0$, we have $|\operatorname{det}(c z+d)|=1$.

Assuming the truth of this lemma, we find that

$$
\left|\operatorname{det}(c z+d)^{-k} e(\operatorname{Tr}(s \gamma \cdot z))\right| \leq|\operatorname{det}(c z+d)|^{-2 g-1} \exp (-2 \pi \operatorname{Tr}(s \operatorname{Im}(\gamma \cdot z)))
$$

for any $k>2 g$, all $z \in U_{g}$ and $\gamma \in \Gamma_{\infty} \backslash \Gamma_{g}$, and also that

$$
\operatorname{det}(c z+d)^{-k} e(\operatorname{Tr}(s \gamma \cdot z)) \longrightarrow 0 \text { as } k \rightarrow+\infty,
$$

for all $z \in U_{g}$ and all $\gamma$ with $c \neq 0$. On the other hand, if $c=0$, we have

$$
\gamma=\left(\begin{array}{cc}
a & 0 \\
0 & t^{-1}
\end{array}\right)
$$

up to $\Gamma_{\infty}$-equivalence, where $a \in \operatorname{GL}(g, \mathbf{Z})$, and hence

$$
\begin{aligned}
\operatorname{det}(c z+d)^{-k} e(\operatorname{Tr}(s \gamma \cdot z)) & =e\left(\operatorname{Tr}\left(s a z^{t} a\right)\right)=e\left(\operatorname{Tr}\left(a z^{t} a s\right)\right) \\
& =e\left(\operatorname{Tr}\left({ }^{t} a s a z\right)\right)=e(\operatorname{Tr}((a \cdot s) z))
\end{aligned}
$$

where $a \cdot s={ }^{t} a s a$ (we use here the fact that $k$ is even).

Using (4), (5) and the absolute convergence of $\mathcal{M}_{s, 2 g+1}(z)$, we find that

$$
\mathcal{P}_{s, k}(z) \longrightarrow \sum_{a \in \mathrm{GL}(g, \mathbf{Z}) / \pm 1} e(\operatorname{Tr}((a \cdot s) z))
$$

as $k \rightarrow+\infty$, for all $z \in U_{g}$. (The series converges as a subseries of the Poincaré series.)

Then we multiply by $e(-\operatorname{Tr}(t z))$ and integrate over $U_{g}$, using (4) and the fact that $\mathcal{M}_{s, 2 g+1}$ is bounded on $U_{g}$ to apply the dominated convergence theorem, and obtain

$$
p_{s, k}(t) \longrightarrow \sum_{a \cdot s=t} 1,
$$

a number that is zero if $s$ and $t$ are not equivalent, or has the same cardinality as $\operatorname{Aut}(s) / 2$ if they are. This completes the proof of Theorem 4.

We still need to prove Lemma 5. We are going to use the description of the Siegel fundamental domain $\mathcal{F}_{g}$ for the action of $\Gamma_{g}$ on $\mathbf{H}_{g}$. Precisely, $\mathcal{F}_{g}$ is the set of $z \in \mathbf{H}_{g}$ satisfying all of the following conditions.

$\dagger$ This statement is used to replace the inequality (1), which has no obvious analogue when $g \geq 2$. 
(1) For all $\gamma \in \Gamma_{g}$, we have

$$
|\operatorname{det}(c z+d)| \geq 1 \text {. }
$$

(2) The imaginary $\operatorname{part} \operatorname{Im}(z)$ is Minkowski-reduced.

(3) The absolute values of all coefficients of $\operatorname{Re}(z)$ are $\leq 1 / 2$.

Siegel showed that the first condition can be weakened to a finite list of inequalities (see, e.g., [3, Proposition 3.3, p. 33]): there exists a finite subset $C_{g} \subset \Gamma_{g}$, such that $z \in \mathbf{H}_{g}$ belongs to $\mathcal{F}_{g}$ if and only if $z$ satisfies (2), (3) and

$$
|\operatorname{det}(c z+d)| \geq 1 \text { for all } \gamma \in C_{g} \text { with } c \neq 0 .
$$

Moreover, if (6) holds with an equality sign for some $\gamma \in C_{g}$, then $z$ is in the boundary of $\mathcal{F}_{g}$; if this is not the case, then $|\operatorname{det}(c z+d)|>1$ for all $\gamma \in \Gamma_{g}$ with $c \neq 0$.

Proof of Lemma 5. First, we show that if $y_{0}>1$ is chosen large enough, the matrix $i y_{0}$ Id is in $\mathcal{F}_{g}$. The only condition that must be checked is (6) when $\gamma \in C_{g}$ satisfies $c \neq 0$, since the other two are immediate (once the definition of Minkowski-reduced is known; it holds for $y_{0} \mathrm{Id}$ when $y_{0} \geq 1$ ). For this, we use the following fact, due to Siegel [8, Lemma 9] (see also [3, Lemma 3.3, p. 34]): for any fixed $z=x+i y \in \mathbf{H}_{g}$ and any $\gamma \in \Gamma_{g}$ with $c \neq 0$, the function

$$
\alpha \mapsto|\operatorname{det}(c(x+i \alpha)+d)|^{2}
$$

is strictly increasing on $[0,+\infty[$ and has limit $+\infty$ as $\alpha \rightarrow+\infty$. Taking $z=i$, we find that

$$
\lim _{y \rightarrow+\infty}|\operatorname{det}(i y c+d)|=+\infty
$$

for every $\gamma \in C_{g}$. In particular, since $C_{g}$ is finite, there exists $y_{0}>1$ such that

$$
\left|\operatorname{det}\left(c z_{0}+d\right)\right|>1
$$

for $z_{0}=i y_{0}$ and $\gamma \in C_{g}$, which is (6) for $i y_{0}$.

Because $\mathcal{U}_{g}$ is compact, it is now also easy to extend this to $z=x+i y_{0}$ with $x \in \mathcal{U}_{g}$. Precisely, for fixed $\gamma \in \Gamma_{g}$ with $c \neq 0$, the function

$$
\left\{\begin{array}{l}
\left.\mathcal{U}_{g} \times\right] 0,+\infty[\rightarrow \mathbf{R} \\
(x, \alpha) \mapsto|\operatorname{det}(c(x+i \alpha)+d)|^{2}
\end{array}\right.
$$

is a polynomial in the variables $(x, \alpha)$. As a polynomial in $\alpha$, as observed by Siegel, it is in fact a polynomial in $\alpha^{2}$ with non-negative coefficients, and it is non-constant because $c \neq 0$. (It is not difficult to check that the degree, as polynomial in $\alpha$, is $2 \operatorname{rank}(c)$.) This explains the limit

$$
\lim _{y \rightarrow+\infty}|\operatorname{det}(c(x+i y)+d)|^{2}=+\infty,
$$

but it shows also that it is uniform over the compact set $\mathcal{U}_{g}$, and over the $\gamma \in C_{g}$ with $c \neq 0$. Therefore we can find $y_{0}$ large enough so that (6) holds for all $z \in U_{g}$, and indeed holds with the strict condition $|\operatorname{det}(c z+d)|>1$ on the right-hand side. By the remark after (6), this means that $z$ is not in the boundary of $\mathcal{F}_{g}$, and hence (3) holds for all $\gamma$ with $c \neq 0$. 
Remark 6. The argument is very clear when $\operatorname{det}(c) \neq 0$ : we write

$$
\begin{aligned}
\operatorname{det}(c(x+i y)+d) & =\operatorname{det}(i y c) \operatorname{det}\left(1-i y^{-1} c^{-1}(c x+d)\right) \\
& =(i y)^{g} \operatorname{det}(c)\left(1+O\left(y^{-1}\right)\right)
\end{aligned}
$$

for fixed $(c, d)$, uniformly for $x \in \mathcal{U}_{g}$.

Remark 7. It would be interesting to know the optimal value of $y_{0}$ in Lemma 5. For $g=1$, any $y_{0}>1$ is suitable. For $g=2$, Gottschling [1, Satz 1] has determined a finite set $C_{2}$ that determines as above the Siegel fundamental domain, consisting of 19 pairs of matrices $(c, d)$; there are four in which $c$ has rank one, and $c$ is the identity for the others. Precisely: for $c$ of rank one, $(c, d)$ belongs to

$$
\left\{\left(\left(\begin{array}{ll}
1 & 0 \\
0 & 0
\end{array}\right),\left(\begin{array}{ll}
0 & 0 \\
0 & 1
\end{array}\right)\right),\left(\left(\begin{array}{ll}
0 & 0 \\
0 & 1
\end{array}\right),\left(\begin{array}{ll}
1 & 0 \\
0 & 0
\end{array}\right)\right),\left(\left(\begin{array}{cc}
1 & -1 \\
0 & 0
\end{array}\right),\left(\begin{array}{rc}
1 & 0 \text { or } 1 \\
-2 & 1
\end{array}\right)\right)\right\}
$$

and for $c$ of rank two, we have $c=1$ and $d$ belongs to

$$
\left\{0,\left(\begin{array}{cc}
s & 0 \\
0 & 0
\end{array}\right),\left(\begin{array}{ll}
0 & 0 \\
0 & s
\end{array}\right),\left(\begin{array}{ll}
s & 0 \\
0 & s
\end{array}\right),\left(\begin{array}{rr}
s & 0 \\
0 & -s
\end{array}\right),\left(\begin{array}{ll}
0 & s \\
s & 0
\end{array}\right),\left(\begin{array}{ll}
s & s \\
s & 0
\end{array}\right),\left(\begin{array}{ll}
0 & s \\
s & s
\end{array}\right)\right\}
$$

where $s \in\{-1,1\}$. It should be possible to deduce a value of $y_{0}$ using this information. Indeed, quick numerical experiments suggest that, as in the case $g=1$, any $y_{0}>1$ would be suitable.

Remark 8. Analogues of Corollary 3 cannot be derived immediately in the setting of Siegel modular forms because the link between Fourier coefficients and Satake parameters is much more involved; the case $g=2$ is considered, together with further applications and quantitative formulations, in [4].

Acknowledgement. Thanks to M. Burger for helpful remarks concerning the geometry of the Siegel fundamental domain.

\section{References}

1. E. Gottschling, Explizite Bestimmung der Randflächen des Fundamentalbereiches der Modulgruppe zweiten Grades. Math. Ann. 138 (1959), 103-124.

2. H. Iwaniec and E. Kowalski, Analytic Number Theory (American Mathematical Society Colloquium Publications 53), American Mathematical Society (Providence, RI, 2004).

3. H. Klingen, Introductory Lectures on Siegel Modular Forms (Cambridge Studies in Advanced Mathematics 20), Cambridge University Press (Cambridge, 1990).

4. E. Kowalski, A. Saha and J. Tsimerman, Local spectral equidistribution for Siegel modular forms and applications. Preprint, 2010, arXiv:1010.3648.

5. H. Maass, Über die Darstellung der Modulformen $n$-ten Grades durch Poincarésche Reihen. Math. Ann. 123 (1951), 125-151.

6. P. Sarnak, Statistical properties of eigenvalues of the Hecke operator. In Analytic Number Theory and Diophantine Problems (Progess in Mathematics 60), Birkhäuser (Boston, MA, 1987), 75-102.

7. J.-P. Serre, Répartition asymptotique des valeurs propres de l'opérateur de Hecke $T_{p}$. J. Amer. Math. Soc. 10 (1997), 75-102.

8. C. L. Siegel, Symplectic geometry. Amer. J. Math. 65 (1943), 1-86;

www.jstor.org/stable/2371774. 
Emmanuel Kowalski,

ETH Zürich - D-MATH,

Rämistrasse 101, 8092 Zürich,

Switzerland

E-mail: kowalski@math.ethz.ch

Jacob Tsimerman,

Princeton University,

Fine Hall, Princeton NJ 08540,

U.S.A.

E-mail: jtsimerm@math.princeton.edu
Abhishek Saha,

ETH Zürich - D-MATH,

Rämistrasse 101, 8092 Zürich,

Switzerland

E-mail: abhishek.saha@math.ethz.ch 